\title{
BIODIVERSIDADE E CONSIDERAÇÕES BIOGEOGRÁFICAS DAS CYANOBACTERIA DE UMA ÁREA DE MANGUEZAL DO ESTADO DE PERNAMBUCO, BRASIL
}

\author{
Luis Henrique Zanini Branco ${ }^{1}$ \\ Ariadne do Nascimento Moura ${ }^{2}$ \\ Adriana Cristina da Silva ${ }^{1}$ \\ Maria do Carmo Bittencourt-Oliveira ${ }^{3}$
}

\section{Recebido em 20/06/2002. Aceito em 03/06/2003}

\begin{abstract}
RESUMO - (Biodiversidade e considerações biogeográficas das Cyanobacteria de uma área de Manguezal do Estado de Pernambuco, Brasil). Manguezais caracterizam-se por apresentarem amplas variações ambientais (principalmente de salinidade, irradiância e teor de oxigênio) e possuírem substrato relativamente instável, o que exige adaptações específicas dos organismos para habitar com sucesso este ambiente. O presente estudo procura, principalmente, contribuir com o conhecimento da biodiversidade, através da realização do levantamento da flora de cianobactérias habitantes de Manguezais do Estado de Pernambuco. Foram realizadas amostragens quinzenais em quatro pontos distintos do Manguezal do rio Paripe, entre agosto e dezembro/1998, totalizando 27 amostras coletadas. A identificação das principais cianobactérias componentes das comunidades revelou a presença de 16 espécies, distribuídas em 11 gêneros, sete famílias e duas ordens. O gênero Phormidium revelou o maior número de espécies (quatro) e alguns táxons não puderam ser identificados em nível específico (Myxohyella sp. e Oscillatoria sp.). Observou-se a dominância de Oscillatoriales sobre as demais, em termos de número de espécies, bem como a ausência de organismos heterocitados, concordando com a tendência constatada em trabalhos similares. Do ponto de vista biogeográfico, a maioria dos táxons observados tem ocorrência registrada em ambientes salobros, ou mesmo marinhos, de outras regiões, demonstrando a ampla distribuição geográfica e a adaptabilidade desses organismos. Algumas espécies encontradas, como Microcoleus chthonoplastes Gomont, são amplamente distribuídas em ambientes marinhos ou salobros de todo o mundo, enquanto outras são limitadas a regiões geográficas mais restritas.
\end{abstract}

Palavras-chave - Cyanobacteria, Mangue, levantamento taxonômico, biogeografia, Pernambuco

1 Instituto de Biociências, Letras e Ciências Exatas, UNESP, Departamento de Zoologia e Botânica, Rua Cristóvão Colombo, 2265, CEP 15054-000, São José do Rio Preto, SP, Brasil (branco@ bot.ibilce.unesp.br)

2 Departamento Biologia, Universidade Federal Rural de Pernambuco, Rua Dom Manoel de Medeiros, s/n, Dois Irmãos, CEP 52171-030, Recife, PE, Brasil (ariadne@ufrpe.br)

3 Universidade de São Paulo, Escola Superior de Agricultura "Luiz de Queiroz", Departamento de Ciências Biológicas, Av. Pádua Dias, 11, CEP 13418-900, Piracicaba, SP, Brasil (mbitt@esalq.usp.br) 


\begin{abstract}
Biodiversity and biogeographical considerations of Cyanobacteria from a Mangrove area of Pernambuco State, Brazil). Mangroves are characterized by their wide environmental variations (mainly of salinity, irradiance and oxygen content) and by the relatively unstable substratum. These conditions demand specific adaptations of the organisms to successfully inhabit this environment. The main objective of the present study was to contribute with the knowledge of the biodiversity, focusing the cyanobacteria flora of some mangrove areas of Pernambuco State. Fortnightly samplings were carried out in four different sites along Paripe River, between August and December/ 1998, totaling 27 collected samples. The identification of the most representative cyanobacteria of the communities revealed the presence of 16 species, distributed in 11 genera, seven families and two orders. The genus Phormidium revealed the highest species number (four) and some taxa could not be identified at specific level (Myxohyella sp. and Oscillatoria sp.). The dominance of Oscillatoriales over other orders, in terms of number of species, was observed as well as the absence of heterocytous organisms, agreeing with the tendency verified in similar studies. Considering biogeographical aspects, most of the found taxa has been recorded in brackish or even marine environments from other areas, evidencing the wide geographical distribution and adaptability of these organisms. Some species found, as Microcoleus chthonoplastes Gomont, are worldwide distributed in marine or brackish habitats, while other taxa are limited to more restricted geographical areas.
\end{abstract}

Key words - Cyanobacteria, Mangrove, taxonomic survey, biogeography, Pernambuco State

\section{Introdução}

Manguezais são ecossistemas litorâneos caracterizados pela alta produtividade secundária e pelo papel fundamental no histórico de vida de inúmeros organismos marinhos. São também importantes quanto à produtividade primária devido à alta diversidade de organismos fotossintetizantes, onde as algas assumem papel relevante.

Embora os Manguezais sejam reconhecidamente ambientes muito ricos, viver nestes ecossistemas implica em grande adaptação fisiológica, seja para fanerógamos, criptógamos ou animais. Isso se deve ao fato de que este ambiente é sujeito a amplas alterações diárias na salinidade, devido à influência da mistura de águas doces e marinhas regulada, por sua vez, pela variação das marés. Além disso, o substrato costuma ser instável (partículas de solo muito finas) com grande variação no volume de água.

Alguns estudos têm sido realizados em Manguezais do mundo com a finalidade do reconhecimento da flora de cianobactérias e/ou de outros grupos de algas (Beanland \& Woelkerling 1982; Dor 1984; Hussain \& Khoja 1993; Khoja 1998; Lamberti et al. 1989; ZhenFen \& Wei-Gang 1997).

No Brasil, os Manguezais têm sido restritamente avaliados quanto à flora de ciano- bactérias, sendo que os estudos disponíveis sobre o tema foram realizados nos estados do Maranhão (Nogueira \& Ferreira-Correia 2001), Pernambuco (Moura 1991; Moura et al. 2000), Rio de Janeiro (Baeta-Neves \& Tribuzi 1992) e São Paulo (Branco et al. 1994, 1996, 1997; Sant'Anna, 1988). Não foram encontrados artigos similares realizados nos demais Estados brasileiros por onde o ecossistema Manguezal se estende.

Com base na tendência atual da sistemática das cianobactérias, acredita-se que as espécies podem distribuir-se por grandes extensões geográficas, entretanto, espera-se que tais táxons ocupem ambientes ecologicamente semelhantes, não sendo mais admitida a existência vulgar de organismos ubíquos (Komárek 1985; 1994). Segundo Hoffmann (1996), a consideração de diversos fatores relacionados à ecofisiologia e capacidades de dispersão, como por exemplo temperatura, distribuição de habitats, dispersão e especiação, podem resultar em explicações possíveis para os diferentes padrões de distribuição geográfica das cianobactérias. Dessa maneira, o conhecimento das cianobactérias de Manguezais de áreas geográficas distintas, além de contribuir para a complementação de dados de biodiversidade, pode também trazer contribuições relevantes à taxonomia e ecologia do grupo. 
Com este trabalho objetivou-se a ampliação do conhecimento florístico das Cyanobacteria em território brasileiro, a comparação entre as floras de localidades distintas com as mesmas características ecológicas e o fornecimento de dados biogeográficos para a sistemática das cianobactérias, procurando contribuir para a consolidação dos conceitos atuais sobre a distribuição das espécies.

\section{Material e métodos}

Os trabalhos de campo foram realizados no Manguezal do rio Paripe (7०41'39'"-748'54' S e 34⒋'12" $-34^{\circ} 53^{\prime} 13^{\prime \prime}$ W), localizado ao sul da Ilha de Itamaracá, distante $50 \mathrm{~km}$ da cidade do Recife, litoral norte do Estado de Pernambuco (Fig. 1). O rio Paripe é o único que nasce na Ilha de Itamaracá, deságua próximo à saída sul do Canal de Santa Cruz e toda sua bacia está localizada na zona da mata do Estado de Pernambuco. O clima é quente e úmido, com dois períodos diferenciados: um seco, entre os meses de setembro e fevereiro, e um chuvoso, entre março e agosto. Esse Manguezal sofre influência constante das águas costeiras do Canal de Santa Cruz. O regime de salinidade na área varia de eualino a oligoalino e não é um ambiente considerado poluído do ponto de vista orgânico (Moura 1991). A área possui solo lamacento e arenoso típico desses ecossistemas, onde a vegetação arbórea é predominantemente composta por Rhizophora mangle L., Avicennia schaueriana Staft \& Leemann e Laguncularia racemosa Gaerth.

Foram escolhidas quatro estações de coleta ao longo da área de Manguezal: uma à montante, uma intermediária e duas à jusante (Fig. 1). Na região da jusante foram estabelecidas duas estações, pois é uma área onde o sedimento caracteriza-se por apresentar manchas escuras de grande extensão, com crescimentos visíveis de cianobactérias.

As coletas de material biológico e de dados foram realizadas quinzenalmente no período de agosto a dezembro/1998 e foram procedidas de maneira aleatória, a partir da visualização das massas algáceas sobre o sedimento, sobre pneumatóforos ou sobre raízes-escoras. As massas foram coletadas com auxílio de espátula e preservadas em solução de formol (4\%). Amostras de macroalgas também foram recolhidas para estudo das cianobactérias epífitas.

No laboratório, as massas foram observadas primeiramente sob microscópio estereoscópico Carl-Zeiss, para caracterização geral. O material a ser estudado foi colocado entre lâmina e lamínula para estudo ao microscópio fotônico Olympus (modelo BX50). Para cada amostra coletada foram montados e examinados, no mínimo, cinco conjuntos. A medição das estruturas utilizadas na identificação taxonômica foi realizada utilizando-se ocular micrometrada de tambor Olympus (modelo OSM4) acoplada ao sistema de oculares do microscópio fotônico. O registro fotográfico dos táxons encontrados foi feito com o emprego do equipamento Olympus PM-20 acoplado ao microscópio fotônico e os desenhos foram elaborados com auxílio de câmara-clara. Neste estudo, foram considerados apenas os componentes mais representativos em termos de abundância, a partir de estimativa visual.

A identificação dos táxons encontrados foi realizada seguindo-se os procedimentos usuais para a taxonomia das cianobactérias (Branco et al. 1996; 1997). Gêneros e espécies foram distribuídos segundo o sistema de classificação em Komárek \& Anagnostidis (1986, 2000 Chroococcales) e Anagnostidis \& Komárek (1988 - Oscillatoriales).

\section{Resultados}

Foram identificadas 16 espécies de cianobactérias, distribuídas em 11 gêneros, sete famílias e duas ordens. 


\section{Ordem Chroococcales}

Família Hydrococcaceae

Myxohyella sp. (Fig. 2-3)

Talo formando regiões pseudoparenquimatosas e pseudofilamentosas; células da região pseudoparenquimatosa irregulares, aproximadamente isodiamétricas, 4,2-5,6 $\mu \mathrm{m}$ compr., 3,1-4,6 4 m diâm.; células da região pseudofilamentosa irregulares, alongadas, 7,0-16,3 $\mu \mathrm{m}$ compr., 1,8-3,2um diâm.; células terminais dos pseudofilamentos geralmente divididas com aspecto dicotômico; conteúdo celular geralmente com 1 a 2 grânulos grandes.

Hábito: epífita em macroalgas (Bostrychia radicans).
Comentário: a população estudada é similar a $M$. papuana Hoffmann, mas difere em relação aos limites métricos do diâmetro e comprimento celular e pela estrutura do talo, que apresenta nítida distribuição radial em Myxohyella sp. e é alongado em M. papuana.

Família Microcystaceae

Aphanothece cf. castagnei (Brébison) Rabenhorst, Flor. Eur. Alg. II: 64, 1865. (Fig. 4)

Colônias arredondadas ou alongadas, 40-50 $\mu \mathrm{m}$ compr., $25-45 \mu \mathrm{m}$ diâm.; células cilíndricas com extremidades arredondadas, alongadas, 4,0-4,5 $\mu \mathrm{m}$ compr., 3,0-3,7 $\mu \mathrm{m}$ diâm., distribuídas próximas umas às outras; conteúdo celular verde-azulado, levemente granulado.
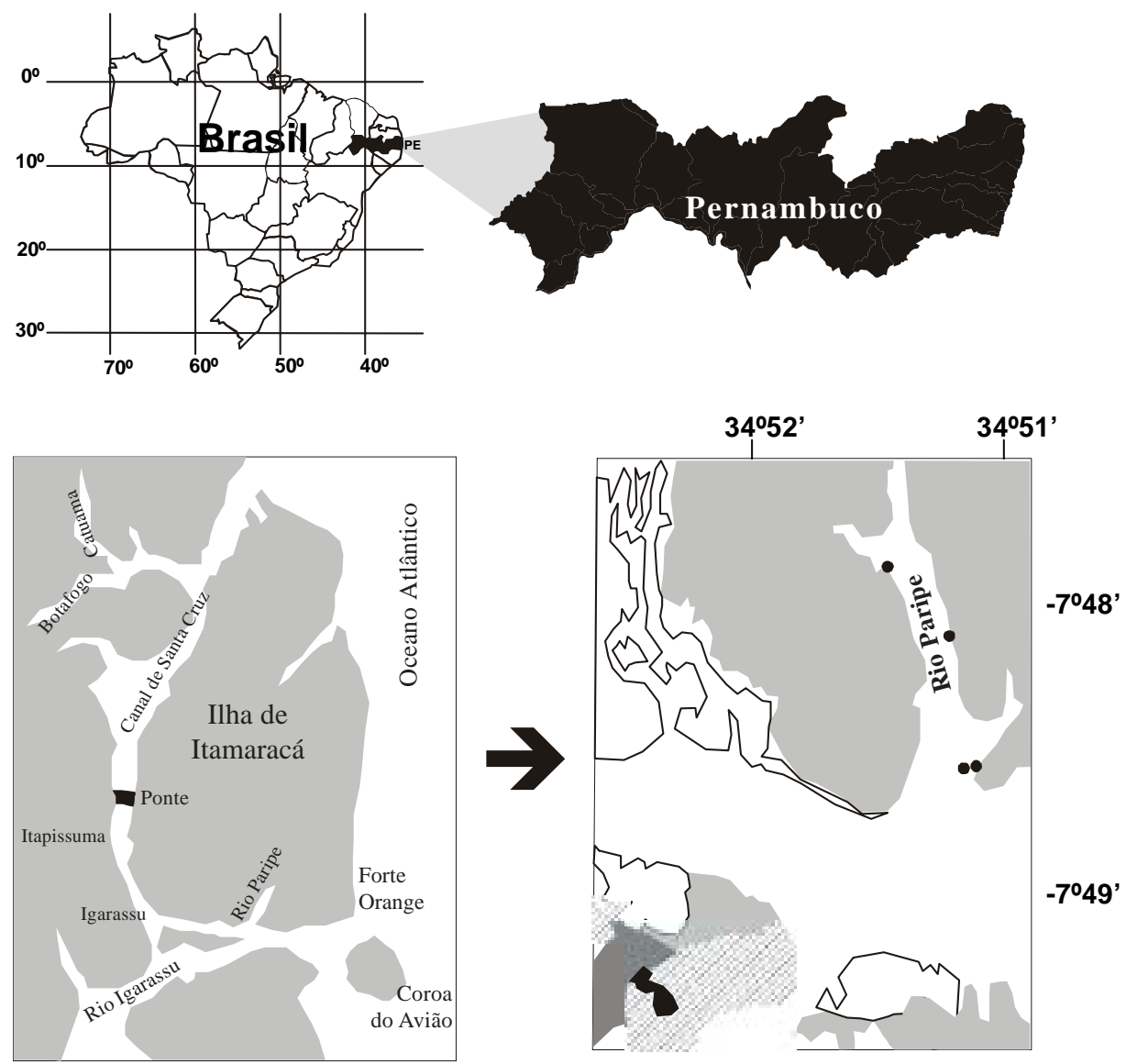

Figura 1. Mapa com a localização da área amostrada no Manguezal do rio Paripe, Recife, PE. 
Hábito: colônias entre outras algas.

Comentário: a população encontrada assemelha-se a A. castagnei em suas características métricas e morfológicas. Entretanto, segundo Komárek \& Anagnostidis (1999), embora provavelmente cosmopolita, a espécie só é encontrada em ambientes úmidos (sobre rochas e entre musgos) e registros de ocorrência em biótopos aquáticos são evidentemente incorretos. Portanto, a diferenciação entre esta população e a espécie refere-se à ecologia dos organismos.

\section{Família Xenococcaceae}

Xenococcus schuboei Thuret, Notes algol. 2: 74-76, 1880. (Fig. 5-6)

Indivíduos unicelulares isolados ou formando colônias densas; células arredondadas, 2,5-8,7 $\mu \mathrm{m}$ diâm., 2,5-10,0 $\mu \mathrm{m}$ compr., contorno poligonal em vista apical; conteúdo celular homogêneo com poucos grânulos grandes; baeócitos não observados.

Hábito: epífita em Microcoleus chthonoplastes.

\section{Ordem Oscillatoriales}

Família Borziaceae

Yonedella lithophila (Ercégovic) Umezaki, Taxon 11(6): 204, 1962. (Fig. 7)

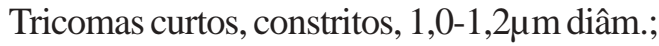
células arredondadas, $0,8-1,2 \mu \mathrm{m}$ compr., relação comprimeto/largura (RC/L) aprox. 1,0; célula apical esférica.

Hábito: tricomas epífitas em outras algas.

Família Oscillatoriaceae

Lyngbya semiplena J. Agardh ex Gomont, Ann. Sci. Nat. 16: 138, 1892. (Fig. 17)

Filamentos 8,9-9,1 $\mu \mathrm{m}$ diâm.; bainha delgada, hialina; tricomas não constritos, não ou muito levemente atenuados em direção ao ápice, 7,6-7,7 $\mu \mathrm{m}$ diâm.; células 1,9-2,8 $\mu \mathrm{m}$ compr., $\mathrm{RC} / \mathrm{L} 0,2$ a 0,4 , septos transversais granulados; conteúdo celular homogêneo; célula apical cônico-arrredondada, espessamento apical presente.

Hábito: filamentos emaranhados entre outras algas (Microcoleus chthonoplastes e Porphyrosiphon martensianus).

\section{Oscillatoria sp. (Fig. 8-10)}

Tricomas levemente constritos, atenuados em direção à extremidade, ápice geralmente

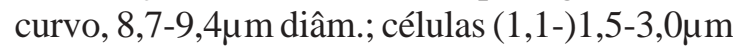
compr., discóides, RC/L 0,1 a 0,3, septos transversais não granulados; conteúdo celular homogêneo; célula apical cônico-arredondada, curta.

Hábito: tricomas isolados, entre outras algas, crescendo sobre substrato arenoso.

Comentários: uma característica comum dos espécimes observados é a presença de extremidades com formas diferentes; devido à frequiente ocorrência de necrídios, muitas células apicais e regiões terminais dos tricomas ainda não se apresentaram diferenciadas, exibindo regiões não afiladas (Fig. 10). Uma observação menos detalhada poderia conduzir a um erro taxonômico. Acredita-se que os indivíduos encontrados pertençam ao gênero Oscillatoria por possuírem células curtas (discóides), embora em alguns casos excepcionais ocorram células pouco mais longas. O conjunto de características dos indivíduos desta população não encontra correspondência satisfatória em nenhuma das espécies descritas nos trabalhos consultados. Phormidium corallinae (Gomont) Anagnostidis \& Komárek foi a espécie considerada mais próxima, mas por apresentar células um pouco mais longas (e por isso é incluída no gênero Phormidium) e espessamento de membrana apical, além de atenuação menos evidente, mostra-se distinta da população encontrada.

Família Phormidiaceae

Microcoleus chthonoplastes Gomont, Ann. Sci. Nat. 15: 353, 1892. (Fig. 11-12)

Massa fina, verde intenso a enegrecida ou filamentos isolados; filamentos com até 12 tricomas, (12-) 21,2-42,2 $\mu \mathrm{m}$ diâm.; bainha hialina, 
geralmente ampla; tricomas constritos, $2,8-3,7 \mu \mathrm{m}$

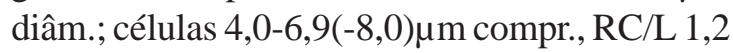
a 2,3, septos transversais não granulados; conteúdo celular homogêneo; célula apical cônico-arredondada, longa.

Hábito: massa de indivíduos crescendo sobre fragmentos de rocha ou filamentos emaranhados entre outras algas.

Microcoleus weeksii Setchell \& Gardner, Univ. Calif. Publ. Bot. 6(17): 470, 1918. (Fig. 13)

Filamentos com até 5 tricomas, 20,0-30,0um diâm.; bainha hialina, ampla; tricomas não constritos, atenuados em direção ao ápice,

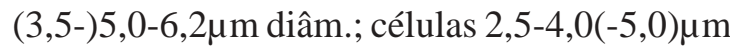
compr., RC/L 0,4 a 0,8(-1,0), septos transversais não granulados; conteúdo celular homogêneo; célula apical cônico-aguda.

Hábito: filamentos crescendo entre outras algas.

Phormidium molle Gomont, Ann. Sci. Nat. 16: 163, 1892. (Fig. 14)

Massa fina, verde escura; filamentos 4,57,0um diâm.; bainha hialina; tricomas constritos, não atenuados em direção ao ápice, $2,5-5,0 \mu \mathrm{m}$ diâm.; células 2,5-6,8 $\mu \mathrm{m}$ compr., geralmente quadráticas a mais longas que largas, ocasionalmente mais curtas que largas, $\mathrm{RC} / \mathrm{L}$ 0,6 a 1,8, septos transversais não granulados; conteúdo celular homogêneo; célula apical cônico-arredondada.

Hábito: massas de indivíduos crescendo sobre o solo.

Phormidium nigro-viride (Thwaites ex Gomont) Anagnostidis \& Komárek, Algolog. Stud., 50-53: 405, 1988. (Fig. 15)

Massa fina, verde escura; tricomas constritos, levemente atenuados em direção ao ápice, raramente curvos na extremidade, 7,0-12,0um diâm.; bainha muito tênue, ocasional; células 2,0-4,0 $\mu$ m compr., RC/L 0,2 a 0,3, septos transversais granulados; conteúdo celular homogêneo; célula apical hemisférica a arredondada, geralmente com espessamento apical.

Hábito: massa de indivíduos crescendo sobre o solo.

Comentário: P. nigro-viride é próxima a $P$. tenue em relação às características métricas, no entanto a presença de atenuação dos tricomas é característica diferencial importante.

Phormidium cf. purpurascens Gomont, Ann. Sci. Nat. 16: 166, 1892. (Fig. 16)

Massa fina, verde oliva; filamentos 2,1$2,7 \mu \mathrm{m}$ diâm.; tricomas não constritos, $2,0-2,5 \mu \mathrm{m}$ diâm.; bainha tênue; células 2,5-3,8 $\mu \mathrm{m}$ compr., quadráticas a mais longas que largas, $\mathrm{RC} / \mathrm{L} 1,0$ a 1,9, septos transversais granulados (1 grânulo em cada lado do septo); conteúdo celular homogêneo; célula apical cilíndrico-arredondada a arredondada.

Hábito: massa de indivíduos crescendo sobre o solo.

Comentário: a presente população possui indivíduos semelhantes aos de P. purpurascens, mas os ambientes descritos para a ocorrência da espécie (subaéreo epilítico e águas termais), bem como a coloração da massa (púrpura) são características distintivas entre as populações em questão.

Phormidium submembranaceum Gomont, Ann. Sci. Nat. 16: 180, 1892. (Fig. 18)

Massa fina, verde escuro a verde oliva; tricomas levemente constritos, levemente atenuados em direção ao ápice, 3,5-4,2 $\mu \mathrm{m}$ diâm.; bainha presente, tênue, hialina; células 3,5-6,2 $\mu \mathrm{m}$ compr., RC/L 0,9 a 1,5, septos transversais não granulados; conteúdo celular homogêneo com poucos grânulos grandes; célula apical cônicoarredondada, calíptra presente.

Hábito: massa de indivíduos crescendo sobre solo.

Porphyrosiphon martensianus (Gomont) Anagnostidis \& Komárek, Algolog. Stud. 50-53: 409, 1988. (Fig. 19) 
Massa fina, verde acastanhada ou filamentos isolados; filamentos 10,0-16,0 $\mathrm{mm}$ diâm.; bainha tênue, hialina; tricomas não constritos, não atenuados em direção ao ápice,

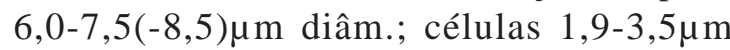
compr., RC/L 0,3 a 0,4, septos transversais granulados; conteúdo celular homogêneo; célula apical arredondada.

Hábito: massa de indivíduos crescendo sobre o solo ou filamentos emaranhados entre outras algas.

Spirulina major Gomont, Ann. Sci. Nat. 16: 251, 1892. (Fig. 20)

Tricomas isolados, 1,5-1,7 $\mu \mathrm{m}$ diâm.; espiras (1,7-)2,0-2,7 $\mu \mathrm{m}$ altura, 2,5-3,7 $\mu \mathrm{m}$ distância; conteúdo celular verde azulado claro, homogêneo.

Hábito: tricomas isolados crescendo entre outras algas.

Família Pseudanabaenaceae

Leptolyngbya lagerheimii (Gomont) Anagnostidis \& Komárek, Algolog. Stud. 50-53: 391, 1988. (Fig. 22)

Tricomas não constritos, 1,2-2,0 $\mu$ m diâm.; bainha ocasional, tênue, hialina; células 1,2$2,5 \mu \mathrm{m}$ compr., geralmente mais curtas que largas a quadráticas, RC/L 0,6 a 1,6, septos transversais não granulados, translúcidos; conteúdo celular homogêneo; célula apical cônico-arredondada.

Hábito: tricomas isolados crescendo entre outras algas.

Leptolyngbya perelegans (Lemmermann) Anagnostidis \& Komárek, Algolog. Stud. 50-53: 392, 1988. (Fig. 21)

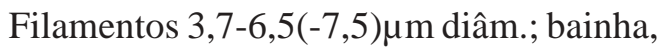
quando presente, de espessura variável, desde tênue até ampla, hialina; tricomas não constritos,

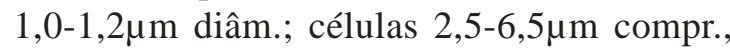
mais longas que largas, RC/L 2,0 a 5,4, septos transversais translúcidos, granulados (2 grânulos em cada lado do septo); conteúdo celular homogêneo; célula apical cilíndrico-arredondada.

Hábito: filamentos isolados crescendo entre outras algas.

\section{Discussão}

Excetuando-se os táxons não identificados em nível específico, Aphanothece cf. castagnei e Phormidium cf. purpurascens, todos os demais já tiveram ocorrências registradas em ambientes de água salobra ou marinha no Brasil.

Em comparação com outros trabalhos de mesma natureza, notou-se que a flora encontrada nos Mangues do rio Paripe tem similaridade com a descrita por Branco et al. $(1996,1997)$ para os Manguezais da Ilha do Cardoso (SP), com nove táxons em comum, correspondendo a $52,9 \%$ encontrados no presente estudo. Por outro lado, os cianobactérias de Mangues do rio Paripe apresentaram apenas três espécies em comum (17,6\% dos táxons encontrados) com os de Manguezais da região de Cabo Frio (RJ), estudados por Baeta-Neves \& Tribuzi (1992). Em comparação com os resultados de Nogueira \& Ferreira-Correia (2001), houve a coincidência de apenas dois táxons. Considerando-se os trabalhos de Moura (1991) e Moura et al. (2000) conjuntamente, apenas cinco táxons $(18,5 \%)$ foram relacionados em comum com a presente avaliação. Tais diferenças entre as floras das diferentes regiões estão possivelmente relacionadas a especificidades na metodologia utilizada para a coleta de material e na abordagem realizada. Acredita-se que as similaridades devem ser mais significativas do que as comparações apresentadas demonstram. A execução de trabalhos com métodos e conceitos padronizados poderia revelar a verdadeira semelhança entre os ambientes das diferentes regiões enfocadas, dadas as características particulares do ecossistema Manguezal.

Embora não tenha sido constatada grande coincidência na composição florística das comunidades das diferentes localidades, em um aspecto mais amplo, os trabalhos demonstram 

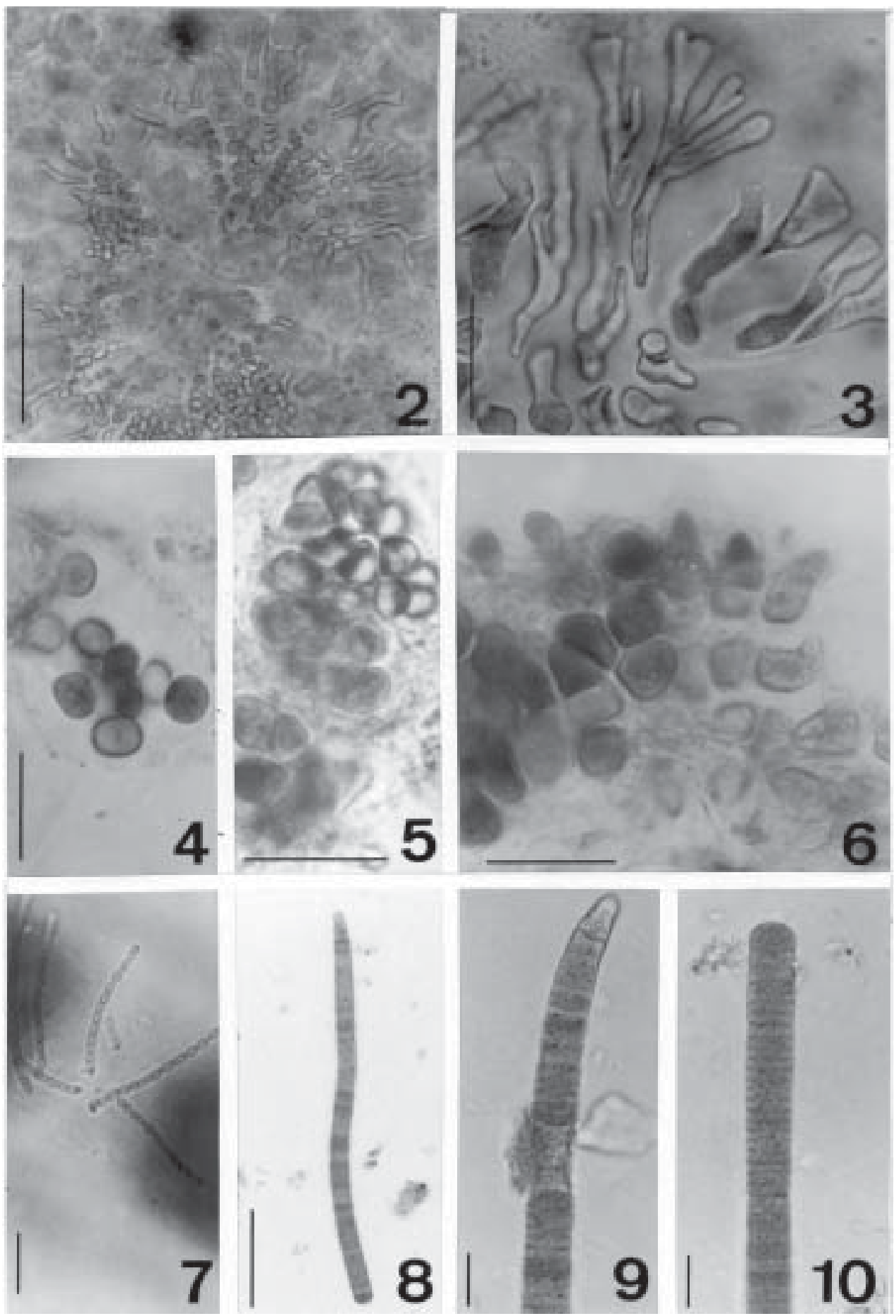

Figuras 2-10. 2-3. Myxohyella sp.; 4. Aphanothece cf. castagnei; 5-6. Xenococcus schubouei; 7. Yonedaella lithophila; 8-10. Oscillatoria sp.; 8. aspecto geral do tricoma mostrando as duas extremidades distintas; 9. tricoma com ápice afilado e formação de necrídio; 10. tricoma com ápice cilíndrico. (escalas: Figuras 2 e $8=50 \mu \mathrm{m}$; Figuras 4-5 = 20 $\mu \mathrm{m}$;

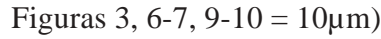



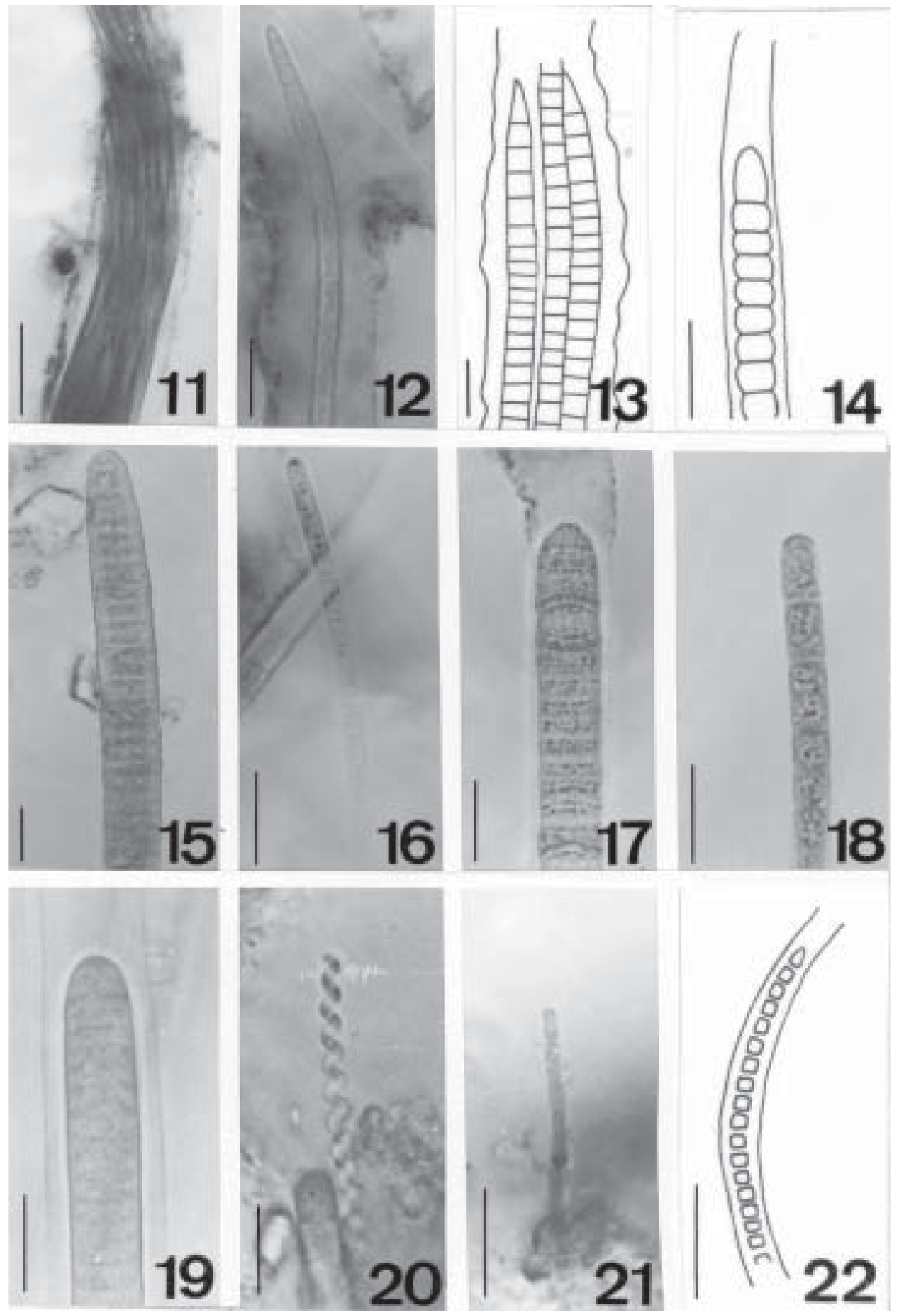

Figuras 11-22. 11-12. Microcoleus chthonoplastes; 13. Microcoleus weeksii; 14. Phormidium molle; 15. Phormidium nigro-viride; 16. Phormidium cf. purpurascens; 17. Lyngbya semiplena; 18. Phormidium submembranaceum; 19. Porphyrosiphon martensianus; 20. Spirulina major; 21. Leptolyngbya pellucida; 22. Leptolyngbya lagerheimii. (escalas: Figura $11=20 \mu \mathrm{m}$; Figuras $12-21=10 \mu \mathrm{m}$ ) 
a predominância das Oscillatoriales sobre as demais ordens de Cyanobacteria (Tab. 1). Isto provavelmente reflete a maior adaptabilidade dos organismos filamentosos homocitados às condições ambientais dos Manguezais.

Entre os táxons encontrados no presente estudo, a grande maioria tem ocorrência registrada para ambientes salobros e marinhos por diversos autores em diversas partes do globo (Carter 1933; Dor 1984; Koster 1960; Lambert et al. 1989; Nogueira \& Ferreira-Correia 2001; Sant'Anna 1995; Sant' Anna \& Simonetti 1992; Sant'Anna et al. 1983; 1985; 1995; Silva 1991). Além das populações não identificadas em nível específico, não foram encontrados na literatura registros de ocorrência de Phormidium cf. purpurascens em ambientes marinhos ou salobros, exigindo cuidados na consideração da real identidade taxonômica destes organismos, bem como na avaliação da distribuição geográfica e ecológica da espécie.

O ecossistema Manguezal torna-se um interessante tema em relação à abordagem taxonômica e à distribuição ecológica das espécies devido à interface dos ambientes de água doce com os de água salgada. Em geral, as floras de cianobactérias de Manguezais revelam maior similaridade com as de ambientes marinhos embora, em tese, possam conter componentes típicos de ecossistemas salobros e de água doce. Segundo Komárek (1994), a idéia de que espécies ubíquas são comuns e de que uma espécie pode ocorrer em habitats muito diferentes nunca foram provadas.

Mesmo que se postule que as espécies apresentam amplitudes ecológicas relativamente estreitas, a limitação ecológica nem sempre implica em limite geográfico restrito (Komárek 1985; 1994). Muitas espécies, que crescem em biótopos que ocorrem em todo o globo, realmente têm distribuição cosmopolita, mas somente em seus biótopos especiais (Komárek 1994). Da mesma maneira, Hoffmann (1994) afirma que, semelhante a outros grupos de cianobactérias, também existem táxons de cianobactérias marinhas com distribuição (sub-)cosmopolita.

A distribuição biogeográfica das espécies de cianobactérias não é tema de fácil abordagem. Aspectos metodológicos, como áreas de amostragem, conceito de espécie utilizado e identificações taxonômicas duvidosas, tornam o trabalho de avaliação biogeográfica pouco preciso (Hoffmann 1994) e, até o momento, restringe-se às espécies mais bem delimitadas (Hoffmann 1994; 1996; 1999).

Entre os táxons encontrados no Manguezal do rio Paripe, Microcoleus chthonoplastes é considerado um organismo cosmopolita. Diversos trabalhos têm registrado a ocorrência da espécie em vários continentes, entretanto quase sempre associada a ambientes salinos (Branco et al. 1996; Frémy 1930; Geitler 1932; Gomont 1892; Hussain \& Khoja 1993; Koster 1960; Lambert et al. 1989; Sant'Anna et al. 1985; Silva 1991). Além das evidências a partir

Tabela 1. Composição de algumas comunidades de cianobactérias em Manguezais segundo a distribuição das espécies nas respectivas ordens [classificação dos táxons nas ordens segundo Anagnostidis \& Komárek (1984; 1990) e Komárek \& Anagnostidis $(1986 ; 1989 ; 2000)]$.

\begin{tabular}{lcccc}
\hline & Chroococcales & Oscillatoriales & Nostocales & Stigonematales \\
\hline Lambert et al. (1991) & 08 & 15 & 04 & 0 \\
Moura (1991); Moura et al. (2000) & 09 & 33 & 0 & 0 \\
Baeta-Neves \& Tribuzi (1992) & 03 & 06 & 02 & 02 \\
Branco (1991); Branco et al. $(1996 ;$ 1997) & 13 & 25 & 07 & 02 \\
Nogueira \& Ferreira-Correia (2001) & 05 & 08 & 02 & 0 \\
Este trabalho & 05 & 12 & 0 & 0 \\
\hline
\end{tabular}


de análises segundo a taxonomia clássica, Garcia-Pichel et al. (1996) comprovaram a ocorrência cosmopolita da espécie através de estudos fenotípicos e filogenéticos, envolvendo técnicas biomoleculares, pela comparação entre culturas de diversas procedências.

É muito provável que outros táxons encontrados tenham ampla área de distribuição, visto que são registrados em diversos trabalhos realizados em ambientes salobros e marinhos de diferentes continentes (Lyngbya semiplena, Microcoleus weeksii, Phormidium nigroviride, Porphyrosiphon martensianus, Spirulina major e Yonedella lithofila). Por outro lado, não se descarta a possibilidade de que alguns táxons tenham distribuição geográfica mais restrita embora, devido ao longo passado evolutivo das cianobactérias e às possibilidades de dispersão nos oceanos, este fato seria surpreendente, a menos que suas taxas evolutivas sejam mais rápidas que as taxas de dispersão (Hoffmann 1994). Devido a suas particularidades taxonômicas, é possível que Myxohyella sp. e Oscillatoria sp. tenham distribuição geográfica restrita, visto que parecem ser táxons distintos dos descritos na literatura especializada.

Em função das informações disponíveis, fica evidente a importância dos levantamentos florísticos dos cianobactérias em diferentes biótopos de regiões tropicais (área ainda pouco explorada), que podem contribuir significativamente para o conhecimento da distribuição geográfica, bem como fornecer subsídios relevantes para a taxonomia destes organismos. Paralelamente, estudos com abordagens moleculares podem também auxiliar no entendimento da biogeografia das Cyanobacteria, cujos membros desempenham papéis ecológicos fundamentais nos diversos ecossistemas onde ocorrem.

\section{Agradecimentos}

Ao CNPq, pela bolsa de Produtividade em Pesquisa ao primeiro Autor e de Iniciação
Científica à terceira Autora; à UFRPE, pela viabilização da execução dos trabalhos de campo.

\section{Referências bibliográficas}

Anagnostidis, K. \& Komárek, J. 1988. Modern approach to the classification system of Cyanophytes. 3 - Oscillatoriales. Algological Studies 50-53: 327-472.

Anagnostidis, K. \& Komárek, J. 1990. Modern approach to the classification system of Cyanophytes. 5 - Stigonematales. Algological Studies 59: 1-73.

Baeta-Neves, M. H. \& Tribuzi, D. 1992. Les Cyanophycées de la mangrove de la "Ponta do Pai Vitório" de la région de Cabo Frio (RJ, Brésil). Acta Biologica Leopoldensia 14: 29-52.

Beanland, W. R. \& Woelkerling, W. J. 1982. Studies on Australian mangrove algae: 2. Composition and geographic distribution of communities in Spencer Gulf, South Australia. Royal Society of Victoria Proceedings 94: 89-106.

Branco, L. H. Z. 1991. Cyanophyceae de comunidades bentônicas do manguezal da Ilha do Cardoso, município de Cananéia, SP. Dissertação de Mestrado. Universidade Estadual Paulista, Rio Claro.

Branco, L. H. Z.; Sant'Anna, C. L.; Azevedo, M. T. P. \& Sormus, L. 1996. Cyanophyte flora from Cardoso Island mangroves, São Paulo State, Brazil. 1. Chroococcales. Algological Studies 80: 101-113.

Branco, L. H. Z.; Sant'Anna, C. L.; Azevedo, M. T. P. \& Sormus, L. 1997. Cyanophyte flora from Cardoso Island mangroves, São Paulo State, Brazil. 2. Oscillatoriales. Algological Studies 84: 39-52.

Branco, L. H. Z.; Silva, S. M. F. \& Sant'Anna, C. L. 1994. Stichosiphon mangle sp. nova, a new Cyanophyte from mangrove environments. Algological Studies 72: 1-7.

Carter, N. 1933. Algal flora of two salt marshes. The Journal of Ecology 21: 128-208.

Dor, I. 1984. Epiphytic blue-green algae (Cyanobacteria) of the Sinai mangal: consideration on vertical zonation and morphological adaptation. Pp. 33-54. In: F. D. Por \& I. Dor (eds.). Hydrobiology of the mangal, Dr. W. Junk Publisher, The Hague.

Frémy, P. 1930. Les Myxophycées de l'Afrique équatoriale française. Archives Botanique Mémoires 3: 1-508. 
Garcia-Pichel, F.; Prufert-Bebout, L. \& Muyzer, G. 1996. Phenotypic and phylogenetic analyses show Microcoleus chthonoplastes to be a cosmopolitan cyanobacterium. Applied and Environmental Microbiology 62: 3284-3291.

Geitler, L. 1932. Cyanophyceae. In: Rabenhorst, L. (ed.). Kryptogamen-flora von Deutschland, Österrech un der Schweiz. Akademische Verlagsgesell., Leipzig.

Gomont, M. M. 1892. Monografie des oscillatoriées (Nostocacées homocystées). Annais du Sciences Naturelles, Botanique, sér. 7, 15: 263-368, 16: 91-264.

Hoffmann, L. 1994. Biogeography of marine bluegreen algae. Algological Studies 75: 137-148.

Hoffmann, L. 1996. Geographic distribution of freshwater blue-green algae. Hydrobiologia 336: 33-39.

Hoffmann, L. 1999. Marine Cyanobacteria in tropical regions: diversity and ecology. European Journal of Phycology 34: 371-379.

Hussain, M. I. \& Khoja, T. M. 1993. Interdital and subtidal blue-green algal mats of open and mangrove areas in the Farasan Archipelago (Saudi Arabia), Red Sea. Botanica Marina 36: 377-388.

Khoja, T. M. 1998. New records of open coast and mangrove algae on the Saudi Coast of the Arabian Gulf. Nova Hedwigia 67: 153-168.

Komárek, J. 1985. Do all Cyanophytes have a cosmopolitan distribution? Survey of the freshwater Cyanophyte flora of Cuba. Algological Studies 71: 359-386.

Komárek, J. 1994. Current trends and species delimitation in the cyanoprokaryote taxonomy. Algological Studies 75: 11-29.

Komárek, J. \& Anagnostidis, K. 1986. Modern approach to the classification system of Cyanophytes. 2- Chroococcales. Algological Studies 43: 157-226.

Komárek, J. \& Anagnostidis, K. 1989. Modern approach to the classification system of Cyanophytes. 4 - Nostocales. Algological Studies 56: $247-345$.

Komárek, J. \& Anagnostidis, K. 2000. Cyanoprokaryota. 1. Teil: Chroococcales. Spektrum, Akademische Verlag (Süßwasserflora von Mitteleuropa; Band 19/1), Berlim.

Koster, J. T. 1960. Caribbean brackish and freshwater Cyanophyceae. Blumea 10: 323-366.
Lambert, G.; Steinke, T. D. \& Naidoo, Y. 1989. Algae associated with mangroves in southern African estuaries: Cyanophyceae. South Africa Journal of Botany 55: 476-491.

Moura, A. N. 1991. Estudo quali-quantitativo das algas perifíticas dos estuários dos rios Paripe e Igarassu-Itamaracá (Pernambuco, Brasil). Dissertação de Mestrado. Universidade Federal Rural de Pernambuco, Recife.

Moura, A. N.; Eskinazi-Leça, E.; Oliveira, G. G.; Pires, A. H. B.; Cavalcante, F. D. \& Silva, M. O. 2000. Diversidade de algas perifíticas em áreas costeiras e estuarinas de Pernambuco. Pp. 1-17. In: Mangrove 2000 - Sustentabilidade de estuários e manguezais. CD-ROM - Trabalhos completos. Recife, ISME-Brasil; UFRPE.

Nogueira, N. M. C. \& Ferreira-Correia, M. M. 2001. Cyanophyceae; Cyanobacteria in red mangrove forest at Mosquitos and Coqueiros estuaries, São Luís, State of Maranhão, Brazil. Revista Brasileira de Biologia 61: 347-356.

Sant'Anna, C. L. 1995. Cyanophyceae marinhas bentônicas do Parque Estadual da Ilha do Cardoso, SP, Brasil. Hoehnea 22: 197-216.

Sant'Anna, C. L. 1988. Scytonemataceae (Cyanophyceae) from the State of São Paulo, southern Brazil. Nova Hedwigia 46: 519-539.

Sant'Anna, C. L.; Azevedo, M. T. P.; Branco, L. H. Z.; Braga, M. R. A.; Cordeiro-Marino, M. \& Guimarães, S. M. P. B. 1995. Cianofíceas marinhas bentônicas das praias de Peruíbe e dos Sonhos, município de Itanhaém, SP, Brasil, III. Revista Brasileira de Biologia 55: 389-407.

Sant'Anna, C. L.; Bicudo, R. M. T. \& Pereira, H. A. S. L. 1983. Nostocophyceae (Cyanophyceae) do Parque Estadual da Ilha do Cardoso, Estado de São Paulo, Brasil. Rickia 10: 1-27.

Sant'Anna, C. L.; Cordeiro-Marino, M.; Braga, M. R. A \& Guimarães, S. M. P. B. 1985. Cianofíceas marinhas bentônicas das praias de Peruíbe e dos Sonhos, município de Itanhaém, SP, Brasil, 1. Rickia 12: 89-112.

Sant'Anna, C. L. \& Simonetti, C. 1992. Cianofíceas marinhas bentônicas das praias de Peruíbe e dos Sonhos, município de Itanhaém, SP, Brasil, II: espécies epilíticas e epizóicas. Revista Brasileira de Biologia 52: 515-523.

Silva, S. M. F. 1991. Flora de Cianofíceas marinhas bentônicas da Ilha da Inhaca, litoral sul de Moçambique, I. Hoehnea 18: 107-125.

Zhen-Fen, L. P. C \& Wei-Gang, L. 1997. Ecological characteristics of macroalgae in mangrove forests in Fujian, China. Acta Botanica Sinica 39: 176-180. 